\title{
Laterally coupled few-electron quantum dots
}

\author{
Andreas Wensauer, Oliver Steffens, Michael Suhrke, and Ulrich Rössler \\ Institut für Theoretische Physik, Universität Regensburg, D-93040 Regensburg, Germany
}

(Version September 15, 2018)

\begin{abstract}
We present ground-state calculations for laterally coupled quantum dots containing 2, 4, and 8 electrons. As our emphasis is on spin effects our results are obtained by applying spin-density functional theory (SDFT). By varying the distance between the centers of the coupled quantum dots, the transition from weak to strong coupling situation is realized. For the 2-electron system we also apply the Heitler-London approximation and analytical concepts to check the reliability of SDFT calculations in this case. In addition we discuss the features of the Coulomb staircase of laterally coupled quantum dots in the weak and strong coupling regimes in comparison to that of a circular parabolic quantum dot.
\end{abstract}




\section{INTRODUCTION}

Semiconductor quantum dots are manmade nanoscale structures in which electrons are confined in all three spatial directions similar to the physical situation in atoms. As they show typical atomic properties like discrete energy levels and shell structures they are often referred to as artificial atomst. However, in contrast to natural atoms, in quantum dots the number of electrons $N$ is tunable and the characteristic lengths of the system corresponding to external confinement potential, electron-electron interaction, and an applied magnetic field are of comparable size. Therefore, these systems are ideal objects to study interaction effects such as transitions in the ground-state spin configuration as a function of the magnetic field.

Starting from quantum dots as a structure which is well understood by now, more complex systems are conceivable and likely to have perspective in future applications, B, A. A simple example is analogous to a two-atom molecule consisting of two coupled quantum dots. The more principal properties of coupled quantum dots follow from the simple model of e.g. two disk-like dots side by side in a plane or on top of each other (lateral or vertical quantum-dot molecules (QDMs)), respectively, which are the subject of experimental5, 6, 0 and theoretica 8, $0,10,11$ studies. Tunneling and overlap of orbital wavefunctions lead to bonding and antibonding states with defined parity, which are delocalized over the two-dot system as in natural systems. The advantage of QDMs is the tunability of the electron number and of the coupling strength by proper design. The latter allows to investigate the transition between weak and strong coupling.

The increased interest in coupled quantum dots is indicated by a growing number of papers on this topic: Austing et al. 0 measured addition spectra of vertical QDM in different coupling regimes. The characteristic features found in their spectra have been confirmed

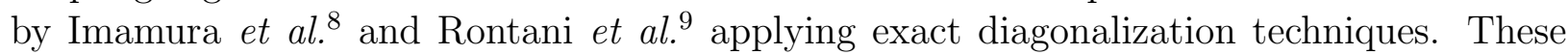
ground-state calculations were performed for up to 6 electrons as a function of the interdot distance and an external magnetic field. Coulomb blockade effects in lateral QDMs have been measured by Waugh et al.6.7, who found a conductance pattern similar to that of single dots for strong coupling, whereas the weakly coupled systems showed a pairing of conductance peaks. Theoretical work on ground-state properties of lateral QDMs was performed by Yannouleas and Landman 10 using an unrestricted Hartree-Fock approach and by Nagaraja et al.11 applying SDFT.

In this paper we investigate the electronic structure of the ground state of two laterally coupled (identical) quantum dots as a function of the interdot distance. In our calculations, applying SDFT, we focus on spin-dependent effects in few-electron quantum dot molecules containing 2, 4, and 8 electrons. In the case of 4 (8) electrons we find a change of spin configuration with decreasing coupling strength from $S_{z}=1\left(S_{z}=1\right)$ to $S_{z}=0\left(S_{z}=2\right)$. For 2 electrons SDFT yields a transition from spin singlet to spin triplet. It turns out, however, that the latter result is an artifact of the SDFT, as can be shown (at least for our choice of the confinement potential) by using alternative approaches. Furthermore, we show the characteristic Coulomb staircases for the chemical potential in the weak and strong coupling limits, respectively.

For all our calculation $\$ 2$ we assume that the two dots are coupled in a quantummechanically coherent way. This means we use delocalized electron states extended over the whole quantum dot molecule whereas states localized to the left or right dot do not 
exist in this description. Note, that this model does not contain all aspects of the actual physical situation where an electron occupying a covalent state interacts with other electrons and charges in the surrounding semiconductor material. These interactions may cause a dephasing of the quantum mechanical wavefunction leading to a breakdown of the delocalized state 13 . Up to now no theory is available which allows a reliable approximation for dephasing rates in realistic nanostructures.

The outline of this paper is the following: In the next section we describe our model of the QDM and discuss the single-particle spectrum of the non-interacting system. In Sec. III we sketch the basics of SDFT and of the numerical method for the simultaneous solution of the Kohn-Sham (KS) equations and calculation of the selfconsistent potentials. The following part is devoted to the 2-electron QDM. We compare the SDFT results with exact (analytical) results and with the Heitler-London approach to point out a particular problem of SDFT. Sec. V relates the spin configuration for 4 and 8 electrons to the single-particle spectrum and summarizes the corresponding SDFT results. Finally we show the typical Coulomb staircase of the addition energies for the weak and the strong coupling limits, respectively.

\section{THE MODEL AND ITS SINGLE-PARTICLE SYSTEM}

We consider quantum dots prepared by laterally confining two-dimensional electrons in a GaAs/AlGaAs heterostructure (material parameters of GaAs: $m^{*}=0.067 m_{e}, \varepsilon=12.4$ ). As the lateral confinement is much weaker than that in growth direction ( $z$-direction) we adopt the standard situation of the electronic quantum limit and describe the electron density by $n(x, y, z)=n(x, y) \delta(z)$. Thus, our system is defined by the effective two-dimensional Hamiltonian

$$
\begin{aligned}
H & =T+V+W \\
& =\sum_{j=1}^{N}\left(\frac{\mathbf{p}_{j}^{2}}{2 m^{*}}+V\left(\mathbf{r}_{j}\right)\right)+\frac{e^{2}}{4 \pi \varepsilon \varepsilon_{0}} \sum_{j=1}^{N} \sum_{k=j+1}^{N} \frac{1}{\left|\mathbf{r}_{j}-\mathbf{r}_{k}\right|}
\end{aligned}
$$

with $\mathbf{p}_{j}$ and $\mathbf{r}_{j}$ being vectors in the $x y$-plane. Our choice of the external confinement potential $V(\mathbf{r})$ is

$$
V(\mathbf{r})=\frac{1}{2} m^{*} \omega_{0}^{2} \min \left((\mathbf{r}-\mathbf{L})^{2},(\mathbf{r}+\mathbf{L})^{2}\right),
$$

where the coupling strength depends on the distance $d=|2 \mathbf{L}|$ between the potential minima at $\pm \mathbf{L}$ in the $x y$-plane (Fig. 1). Along the line between these minima there is a barrier of height

$$
V_{\mathrm{B}}(d)=\frac{1}{2} m^{*} \omega_{0}^{2}\left(\frac{d}{2}\right)^{2} .
$$

This model potential describes a single parabolic quantum dot for $d=0$ as the extreme case of strong coupling and two separate quantum dots of the same shape in the weak-coupling limit for $d \rightarrow \infty$. Due to the broken axial symmetry of $V(\mathbf{r})$ for $d \neq 0$ the numerical requirements are increased in comparison with those for a single circular quantum dot.

In Fig. 2 we show the lowest energy levels of the noninteracting electrons in dependence on the distance between the dot centers for the typical confinement energy $\hbar \omega_{0}=3 \mathrm{meV}$. 
For $d=0$, when the confinement potential of the QDM degenerates to the simple parabolic potential with axial symmetry, the spectrum shows the typical shell structure of an isotropic harmonic oscillator. For large distances $d$ we obtain the same spectrum, now twofold because the system consists of two identical but completely separated quantum dots. By switching on the coupling (or lowering $d$ starting from the weak-coupling limit) we recover the anticipated properties of a diatomic molecule: the energies decrease (increase) with respect to the reference level due to formation of bonding (antibonding) states, which have even (odd) parity as indicated by solid (dashed) lines. The intermediate coupling regime is dominated by crossings leading to the rearrangement of the level structure. In the weak-coupling limit the nth bundle of the twofold level structure with energy $E \approx n \hbar \omega_{0}$ consists of $n$ bonding states and $n$ antibonding states.

\section{SDFT AND KS EQUATIONS}

In order to include the electron-electron interaction we employ the spin-density functional theory (SDFT). It is the generalization of the DFT formalism, originally established by

Hohenberg, Kohn, and Sham $\$ 4,15$, to spin-polarized systems 16 by including the coupling of the magnetization to an applied magnetic field. Accordingly, the Hohenberg-Kohn (HK) theorem has to be modified with respect to the spin degrees of freedom 16 . For this case, it states that two different nondegenerate ground-state wavefunctions $|\Psi\rangle$ and $\left|\Psi^{\prime}\right\rangle$ always yield different tupels $(n(\mathbf{r}), \mathbf{m}(\mathbf{r})) \neq\left(n^{\prime}(\mathbf{r}), \mathbf{m}^{\prime}(\mathbf{r})\right)$ of electron density $n(\mathbf{r})$ and magnetization $\mathbf{m}(\mathbf{r})$. This is sufficient to establish a functional of the total energy with the usual functional properties

$$
E_{V_{0}, \mathbf{B}_{0}}[n, \mathbf{m}]=F_{\mathrm{HK}}[n, \mathbf{m}]+\int \mathrm{d} \mathbf{r}[V(\mathbf{r}) n(\mathbf{r})-\mathbf{B}(\mathbf{r}) \cdot \mathbf{m}(\mathbf{r})]
$$

and the universal HK functional

$$
F_{\mathrm{HK}}[n, \mathbf{m}]=\langle\Psi[n, \mathbf{m}]|T+W| \Psi[n, \mathbf{m}]\rangle .
$$

In the limit $\mathbf{B} \rightarrow 0$ considered here, the SDFT scheme can yield a spin-polarized ground state for even electron numbers due to Hund's rule 17,18. These effects have already attracted much interest and will also be discussed in this paper.

The spin-degree of freedom is considered in the Kohn-Sham (KS) equations 16 by assuming the total spin $S_{z}$ in $z$-direction to be a good quantum number

$$
\begin{array}{r}
\left\{-\frac{\hbar^{2}}{2 m^{*}} \nabla^{2}+V(\mathbf{r})+\frac{e^{2}}{4 \pi \varepsilon \varepsilon_{0}} \int \mathrm{d} \mathbf{r}^{\prime} \frac{n\left(\mathbf{r}^{\prime}\right)}{\left|\mathbf{r}-\mathbf{r}^{\prime}\right|}\right. \\
\left.+V_{\mathrm{XC}}^{\sigma}\left(\left[n_{+}, n_{-}\right], \mathbf{r}\right]\right\} \varphi_{j}^{\sigma}(\mathbf{r})=\varepsilon_{j}^{\sigma} \varphi_{j}^{\sigma}(\mathbf{r})
\end{array}
$$

with the spin $\sigma= \pm$ in $z$-direction and the $\mathrm{KS}$ energies $\varepsilon_{1}^{\sigma} \leq \varepsilon_{2}^{\sigma} \leq \ldots$. For a system containing $N$ particles we calculate the occupation numbers of the KS levels in the ground state due to

$$
\begin{aligned}
\gamma_{j}^{\sigma}=1 & \varepsilon_{j}<\mu \\
0 \leq \gamma_{j}^{\sigma} \leq 1 & \varepsilon_{j}=\mu \\
\gamma_{j}^{\sigma}=0 & \varepsilon_{j}>\mu
\end{aligned}
$$


( $\mu$ : chemical potential) with the constraints

$$
\begin{aligned}
\sum_{j} \gamma_{j}^{\sigma} & =N^{\sigma} \\
N^{+}+N^{-} & =N .
\end{aligned}
$$

This leads to the spin densities

$$
n_{\sigma}(\mathbf{r})=\sum_{j} \gamma_{j}^{\sigma}\left|\varphi_{j}^{\sigma}(\mathbf{r})\right|^{2}
$$

the total density

$$
n(\mathbf{r})=n_{+}(\mathbf{r})+n_{-}(\mathbf{r})
$$

and the magnetization in $z$-direction $\left(\mu_{B}\right.$ : Bohr's magneton)

$$
m_{z}(\mathbf{r})=-\mu_{\mathrm{B}}\left(n_{+}(\mathbf{r})-n_{-}(\mathbf{r})\right) .
$$

The exchange-correlation (xc) potentials

$$
V_{\mathrm{XC}}^{\sigma}\left(\left[n_{+}, n_{-}\right], \mathbf{r}\right)=\frac{\delta E_{\mathrm{XC}}\left[n_{+}, n_{-}\right]}{\delta n_{\sigma}(\mathbf{r})}
$$

are defined as functional derivatives of the xc energy functional

$$
\begin{aligned}
E_{\mathrm{XC}}\left[n_{+}, n_{-}\right]= & F_{\mathrm{HK}}\left[n_{+}, n_{-}\right]-\frac{1}{2} \frac{e^{2}}{4 \pi \varepsilon \varepsilon_{0}} \int \mathrm{d} \mathbf{r} \int \mathrm{d} \mathbf{r}^{\prime} \frac{n(\mathbf{r}) n\left(\mathbf{r}^{\prime}\right)}{\left|\mathbf{r}-\mathbf{r}^{\prime}\right|} \\
& -T_{\mathrm{S}}\left[n_{+}, n_{-}\right] .
\end{aligned}
$$

$\left(T_{\mathrm{S}}\left[n_{+}, n_{-}\right]\right.$denotes the kinetic energy functional of the KS system.) The total ground-state energy $E_{0}$ of the interacting system can be calculated from

$$
\begin{aligned}
E_{0}= & \sum_{j, \sigma} \gamma_{j}^{\sigma} \varepsilon_{j}^{\sigma}-\frac{1}{2} \frac{e^{2}}{4 \pi \varepsilon \varepsilon_{0}} \int \mathrm{d} \mathbf{r} \int \mathrm{d} \mathbf{r}^{\prime} \frac{n(\mathbf{r}) n\left(\mathbf{r}^{\prime}\right)}{\left|\mathbf{r}-\mathbf{r}^{\prime}\right|} \\
& +E_{\mathrm{XC}}\left[n_{+}, n_{-}\right]-\sum_{\sigma} \int \mathrm{d} \mathbf{r} V_{\mathrm{XC}}^{\sigma}\left(\left[n_{+}, n_{-}\right], \mathbf{r}\right) n_{\sigma}(\mathbf{r}) .
\end{aligned}
$$

Concerning the xc potentials we apply the local spin density approximation (LSDA)

$$
E_{\mathrm{XC}}\left[n_{+}, n_{-}\right] \approx \int \mathrm{d} \mathbf{r}\left(n_{+}(\mathbf{r})+n_{-}(\mathbf{r})\right) \varepsilon_{\mathrm{XC}}\left(n_{+}(\mathbf{r}), n_{-}(\mathbf{r})\right)
$$

and the Padé approximation of $\varepsilon_{\mathrm{XC}}\left(n_{+}(\mathbf{r}), n_{-}(\mathbf{r})\right)$ in two dimensions following Tanatar and Ceperleyt 19 .

For the numerical solution of the KS equations we calculate all quantities with spatial dependence on a two-dimensional grid in real space. The damped gradient iteration 20 ensures a simultaneous solution of the KS equations together with the corresponding self-consistent potentials. This method uses the iteration scheme

$$
\left|\Psi^{(k+1)}\right\rangle=H^{-1}\left|\Psi^{(k)}\right\rangle
$$


with the KS Hamiltonian $H$ (only positive eigenvalues), which converges to the ground-state wavefunction. The inversion of the Hamiltonian is performed approximately 20 , 11 using

$$
\begin{aligned}
\left|\Psi^{(k+1)}\right\rangle \approx & \left\{\left[1-\tilde{x}_{0}\left(\frac{p_{x}^{2}}{2 m^{*}}+V_{x}^{\infty}+E_{x 0}\right)^{-1} .\right.\right. \\
& \cdot\left(\frac{p_{y}^{2}}{2 m^{*}}+V_{y}^{\infty}+E_{y 0}\right)^{-1} \cdot \\
& \left.\left.\cdot\left(H-\left\langle\Psi^{(k)}|H| \Psi^{(k)}\right\rangle\right)\right]\right\}\left|\Psi^{(k)}\right\rangle
\end{aligned}
$$

instead of Eq. (19), where $\tilde{x}_{0}, E_{x 0}, E_{y 0}$ are iteration parameters and $V_{x}^{\infty}, V_{y}^{\infty}$ the asymptotic contributions of the external potential for $|\mathbf{r}| \rightarrow \infty$. Excited state KS wavefunctions are calculated using the same iteration scheme with an additional orthogonalization routine. The Hartree and xc potentials depending on the densities of the KS wavefunctions are recalculated in each step. Thus, the KS Hamiltonian is modified in each step of the iteration.

\section{RESULTS FOR THE TWO-ELECTRON QDM}

Fig. 3 depicts the KS energies for a quantum dot molecule containing two electrons as a function of the distance $d$ between the centers of the molecule (we assume $\hbar \omega_{0}=3 \mathrm{meV}$ ).

Starting with $d=0$ the QDM degenerates into a circular parabolic quantum dot showing the known shell structure of the noninteracting case. Due to the closed shell for 2 electrons the ground state is not spin-polarized. This ground-state spin configuration is stable for increasing $d$ as long as the energy gap between the levels (a) and (b) in the single-particle spectrum (Fig. 2) is not too small. At $d \approx 4.5 \mathrm{a}_{0}^{*}\left(\mathrm{a}_{0}^{*}=9.79 \mathrm{~nm}\right.$ denotes the effective Bohr's radius for GaAs) the unpolarized ground state changes into a polarized one. This state with aligned spins persists as SDFT ground state even for $d \rightarrow \infty$, because the energy gap between states (a) and (b) continues to decrease with increasing $d$. This result, similar

to that obtained e.g. by Nagaraja et al.11, differs from those of the physically analogous problems of the vertical QDMs, 22 and from the hydrogen molecule whose ground states are spin singlets.

The SDFT result is also in contrast to a mathematical theorem stating that the spin configuration of the ground state of a two-electron quantum dot molecule, with our definition of the external confinement potential, should be a spin singlet state: As the total Hamiltonian $H$, Eq. (1), does not depend on spin coordinates we can focus on the spatial wavefunctions. Due to Theorem XIII.47 of Ref. 23 the (spatial) ground state wavefunction of the considered two-body-problem is positive and nondegenerate, this means it is symmetric in the spatial coordinates. As a consequence of Pauli's principle the ground state has to be a spin singlet state.

In view of this rigorous statement, the SDFT results of Fig. 3 for $d \geq 4.5 \mathrm{a}_{0}^{*}$ cannot be considered as true ground state values (the same holds for the corresponding SDFT densities).

We test this finding by using a perturbation approach analogous to the Heitler-London model for the hydrogen molecule, which in contrast to SDFT has the advantage of yielding an upper limit for the ground state energy. Similar calculations for other shapes of the external potential were done in Ref. 4 . 
For the two-electron QDM we rewrite the total Hamiltonian (便) as

$$
\begin{aligned}
H= & \frac{\mathbf{p}_{1}^{2}}{2 m^{*}}+\frac{1}{2} m^{*} \omega_{0}^{2} \min \left(\left(\mathbf{r}_{1}-\mathbf{L}\right)^{2},\left(\mathbf{r}_{1}+\mathbf{L}\right)^{2}\right) \\
& \frac{\mathbf{p}_{2}^{2}}{2 m^{*}}+\frac{1}{2} m^{*} \omega_{0}^{2} \min \left(\left(\mathbf{r}_{2}-\mathbf{L}\right)^{2},\left(\mathbf{r}_{2}+\mathbf{L}\right)^{2}\right) \\
& +\frac{e^{2}}{4 \pi \varepsilon \varepsilon_{0}} \frac{1}{\left|\mathbf{r}_{1}-\mathbf{r}_{2}\right|} \\
= & H_{1}^{\mathrm{R}}+H_{2}^{\mathrm{L}}+U_{1}^{\mathrm{R}}+U_{2}^{\mathrm{L}}+W
\end{aligned}
$$

with

$$
\begin{aligned}
H_{j}^{\mathrm{R} / \mathrm{L}} & =\frac{\mathbf{p}_{j}^{2}}{2 m^{*}}+\frac{1}{2} m^{*} \omega_{0}^{2}\left(\mathbf{r}_{j} \mp \mathbf{L}\right)^{2} \\
U_{j}^{\mathrm{R} / \mathrm{L}} & =\frac{1}{2} m^{*} \omega_{0}^{2} \min \left(0, \pm 4 \mathbf{r}_{j} \cdot \mathbf{L}\right) \\
W & =\frac{e^{2}}{4 \pi \varepsilon \varepsilon_{0}} \frac{1}{\left|\mathbf{r}_{1}-\mathbf{r}_{2}\right|}
\end{aligned}
$$

The ground-state wavefunctions of the shifted harmonic oscillators $H^{\mathrm{R} / \mathrm{L}}$ are denoted by $\left|\Psi^{\mathrm{R} / \mathrm{L}}\right\rangle$. So we can use the typical Heitler-London ansatz for the (unnormalized) singlet $\left|\Psi^{+}\right\rangle$and triplet $\left|\Psi^{-}\right\rangle$spatial wavefunctions

$$
\left|\Psi^{ \pm}\right\rangle=\frac{1}{\sqrt{2}}\left(\left|\Psi^{\mathrm{R}}\right\rangle^{(1)}\left|\Psi^{\mathrm{L}}\right\rangle^{(2)} \pm\left|\Psi^{\mathrm{R}}\right\rangle^{(2)}\left|\Psi^{\mathrm{L}}\right\rangle^{(1)}\right)
$$

to calculate the expectation value of the ground-state energy of the system

$$
\begin{aligned}
E_{\mathrm{HL}}^{ \pm}= & \frac{\left\langle\Psi^{ \pm}|H| \Psi^{ \pm}\right\rangle}{\left\langle\Psi^{ \pm} \mid \Psi^{ \pm}\right\rangle}= \\
= & 2 \hbar \omega_{0}+\frac{\left\langle\Psi^{\mathrm{R}}\left|U^{\mathrm{R}}\right| \Psi^{\mathrm{R}}\right\rangle+\left\langle\Psi^{\mathrm{L}}\left|U^{\mathrm{L}}\right| \Psi^{\mathrm{L}}\right\rangle}{1 \pm \mathrm{e}^{-2 L^{2} / l_{h}^{2}}} \\
& +\frac{{ }^{(1)}\left\langle\left.\Psi^{\mathrm{R}}\right|^{(2)}\left\langle\Psi^{\mathrm{L}}|W| \Psi^{\mathrm{R}}\right\rangle^{(1)} \mid \Psi^{\mathrm{L}}\right\rangle^{(2)}}{1 \pm \mathrm{e}^{-2 L^{2} / l_{h}^{2}}} \\
& \pm \frac{\mathrm{e}^{-L^{2} / l_{h}^{2}}\left(\left\langle\Psi^{\mathrm{R}}\left|U^{\mathrm{R}}\right| \Psi^{\mathrm{R}}\right\rangle+\left\langle\Psi^{\mathrm{L}}\left|U^{\mathrm{L}}\right| \Psi^{\mathrm{L}}\right\rangle\right)}{1 \pm \mathrm{e}^{-2 L^{2} / l_{h}^{2}}} \\
& \pm \frac{{ }^{(1)}\left\langle\left.\Psi^{\mathrm{R}}\right|^{(2)}\left\langle\Psi^{\mathrm{L}}|W| \Psi^{\mathrm{L}}\right\rangle^{(1)} \mid \Psi^{\mathrm{R}}\right\rangle^{(2)}}{1 \pm \mathrm{e}^{-2 L^{2} / l_{h}^{2}}} .
\end{aligned}
$$

Note that all matrix elements are real quantities. $l_{h}=\sqrt{\hbar / m^{*} \omega_{0}}$ denotes the characteristic oscillator length. The result for the matrix elements is

$$
\begin{aligned}
& \left\langle\Psi^{\mathrm{R}}\left|U^{\mathrm{R}}\right| \Psi^{\mathrm{R}}\right\rangle+\left\langle\Psi^{\mathrm{L}}\left|U^{\mathrm{L}}\right| \Psi^{\mathrm{L}}\right\rangle= \\
& =2 \hbar \omega_{0}\left(-\frac{L}{l_{h} \sqrt{\pi}} \mathrm{e}^{-L^{2} / l_{h}^{2}}+\frac{L^{2}}{l_{h}^{2}}\left(1-\operatorname{erf}\left(L / l_{h}\right)\right)\right) \\
& { }^{(1)}\left\langle\left.\Psi^{\mathrm{R}}\right|^{(2)}\left\langle\Psi^{\mathrm{L}}|W| \Psi^{\mathrm{R}}\right\rangle^{(1)} \mid \Psi^{\mathrm{L}}\right\rangle^{(2)}= \\
& =\hbar \omega_{0} \sqrt{\frac{\pi}{2}} \frac{l_{h}}{\mathrm{a}_{0}^{*}} \mathrm{e}^{-L^{2} / l_{h}^{2}} \mathrm{I}_{0}\left(L^{2} / l_{h}^{2}\right)
\end{aligned}
$$




$$
\begin{gathered}
\left\langle\Psi^{\mathrm{R}}\left|U^{\mathrm{R}}\right| \Psi^{\mathrm{L}}\right\rangle+\left\langle\Psi^{\mathrm{L}}\left|U^{\mathrm{L}}\right| \Psi^{\mathrm{R}}\right\rangle=-2 \hbar \omega_{0} \frac{L}{l_{h} \sqrt{\pi}} \mathrm{e}^{-L^{2} / l_{h}^{2}} \\
{ }^{(1)}\left\langle\left.\Psi^{\mathrm{R}}\right|^{(2)}\left\langle\Psi^{\mathrm{L}}|W| \Psi^{\mathrm{L}}\right\rangle^{(1)} \mid \Psi^{\mathrm{R}}\right\rangle^{(2)}=\hbar \omega_{0} \sqrt{\frac{\pi}{2}} \frac{l_{h}}{\mathrm{a}_{0}^{*}} \mathrm{e}^{-2 L^{2} / l_{h}^{2}}
\end{gathered}
$$

with the modified Bessel's function $\mathrm{I}_{0}(x)$ and the error function erf $(x)^{24}$.

In Fig. 1 we compare the ground-state energies resulting from SDFT calculations with the Heitler-London approach. For $0 \leq d \leq 4.5 \mathrm{a}_{0}^{*}$ the SDFT singlet ground-state energies are lower than the HL energies. As the comparison between SDFT and exact diagonalization for 2-electron quantum dots shows good agreemente2s, we expect this to hold also for the QDM with small $d$. Moreover, the HL triplet energy diverges for $d \rightarrow 0$ and becomes larger than the HL singlet energy. Therefore, we regard the SDFT results for small $d$ as a better approximation of the ground state than the HL resultt 26 .

If, however, $d$ is increased beyond $4.5 \mathrm{a}_{0}^{*}$ the HL energies become lower than the SDFT results and replace them as ground-state energies. It can be shown analytically that for sufficiently large $d$ the $\mathrm{HL}$ energies fulfill the relation $E_{\mathrm{HL}}^{+}<E_{\mathrm{HL}}^{-}$. Thus, in the limit $d \rightarrow \infty$ the HL results are consistent with the mathematical theorem quoted above. In addition, in the weak-coupling limit the interaction is reduced to the classical Coulomb repulsion between point charges and the total energy approaches asymptotically $E=2 \hbar \omega_{0}+e^{2} /\left(4 \pi \varepsilon \varepsilon_{0} d\right)$. This is also consistent with the energies of the HL approach.

The energy difference of $0.36 \mathrm{meV}$ between $E_{\mathrm{SDFT}}^{-}$and $E_{\mathrm{HL}}^{+}$in the weak-coupling limit can be ascribed to the self-interaction within the SDFT scheme: The charge density of a weakly coupled quantum dot molecule is mainly localized in its centers (charge $1 e^{-}$per center). However, within the SDFT formalism the energy of each charge in its own Coulomb field is counted additionally leading to an enhanced total energy 27. To illustrate this, let us to apply the SDFT concept to a single-particle problem. For a circular parabolic quantum dot with $\hbar \omega_{0}=3 \mathrm{meV}$ this gives a ground-state energy of $3.18 \mathrm{meV}$, i.e. the self-interaction error is $0.18 \mathrm{meV}$. This value has to be doubled for the $d \rightarrow \infty$ limit of the QDM. Thus, we identify the difference $E_{\mathrm{SDFT}}^{-}-E_{\mathrm{HL}}^{ \pm}$as the self-interaction energy in this limit.

The energy difference between the singlet state and the triplet state in the SDFT approach can be qualitatively explained as an xc effect: Both spin configurations show similar total electron densities. Consequently the contributions from the Hartree energy and the spin-independent xc energy can be taken to be approximately equal for singlet and triplet states. However, the triplet total energy is in addition lowered by spin-dependent xc effects

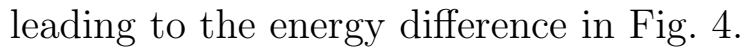

To summarize this section: for small $d\left(0 \leq d \leq 4.5 \mathrm{a}_{0}^{*}\right)$ we find the SDFT (singlet) energies to be the better approximation to the ground state than the HL results, while for larger $d\left(d \geq 4.5 \mathrm{a}_{0}^{*}\right)$ the SDFT energies become too large due to the self-interaction error and the HL results provide the better approximation of the ground-state energy.

\section{RESULTS FOR QDMS CONTAINING 4 AND 8 ELECTRONS}

In this section we present our results for the ground-state energy and spin-configuration for artificial molecules containing 4 and 8 electrons as a function of the interdot distance 
d. Our calculations show that a spin-polarized ground state is accompanied by a (quasi)degeneracy at the Fermi level in the noninteracting single-particle spectrum whereas unpolarized ground states require a sufficiently large gap between the highest occupied and lowest unoccupied single-particle levels of the noninteracting spectrum.

Single dots with 4 electrons have a spin-polarized ground state $\left(S_{z}=1\right)$ due to Hund's ruld17.18. This is the case also for the QDM with $d=0$ (Fig. 5). The spin-polarized ground state persists with increasing $d$ as long as the xc energy overcompensates the energy costs of putting electrons with parallel spins into the bonding and antibonding states ((b) and (c) in Fig. 2) evolving from the second level at $d=0$. For $d>2 \mathrm{a}_{0}^{*}$ it becomes energetically more favorable to occupy both the bonding level (a) and antibonding level (b) (Fig. 2) with two particles forming an $S_{z}=0$ ground state. This configuration remains stable even in the weak coupling limit and reflects the noninteracting particle picture (Fig. 2) which does not show level crossings at the Fermi energy for $N=4$ and large $d$. This result is in agreement with the work of Nagaraja et al.11 (laterally coupled dots), Rontani et al. $\mathbf{0}$, and Imamura et al. (vertically coupled dots). On the other hand, Nagaraja et al.11 do not find a spin-polarized ground state in the strong coupling limit, because their confinement potential obtained by simulating external gates does not approach that of a circular parabolic dot as in our case.

Although even in the limit of large $d$ a quantum-mechanical coupling (delocalized bonding and antibonding states) between the dots can be observed within the SDFT scheme the resulting density (Fig. 6) and energy (Fig. 5) tend towards a semiclassical picture: the densities of the dots are well separated and the total energy of the two coupled dots consists of twice the (quantum-mechanical) energy of a single dot containing 2 electrons and the energy of classical repulsion of two point-like charges $\left(2 e^{-}\right)$in the distance $d$ (see inset of Fig. 5). In contrast to the 2-electron QDM, this test for the asymptotics of the total energy being fulfilled underlines the reliabilty of the SDFT results for 4 electrons.

Turning to QDMs with 8 electrons (Fig. 1) we find for $d=0$ the expected spin-polarized ground state $\left(S_{z}=1\right)$ 17, 18. Increasing $d$ up to $7.5 \mathrm{a}_{0}^{*}$ this state persists with double-occupancy of the levels (a), (b), and (c) of Fig. 2 and single-occupancy of the quasi-degenerate levels (d) and (e) with aligned spins. At $d \approx 7.5 \mathrm{a}_{0}^{*}$ the levels (c), (d), (e), and (f) of Fig. 2 get close enough that an alignment of all four spins at the Fermi level can lower the total energy. Therefore, we find a spin-polarized ground state in the weak coupling regime $\left(d>8 \mathrm{a}_{0}^{*}\right)$ in agreement with Nagaraja et al.11. Although the spin-aligned ground state was ruled out in the case of 2 electrons we believe that the $S_{z}=2$ ground state for the 8-electron artificial molecule in the weak-coupling regime is reliable. This is emphasized by the correct asymptotics of the total energy in the limit $d \rightarrow \infty$ (see inset of Fig. 7). Exact diagonalizations 8 , 8 for vertically coupled dots performed for up to 6 electrons yield a spinpolarized ground state $\left(S_{z}=1\right)$ for the 6-electron artificial molecule in the weak coupling regime, too, indicating that spin-polarized ground states can exist in principle for QDMs.

Also for $N=8$, in the weak-coupling limit, the total energy and density can be understood in a semiclassical picture: the densities (for sufficiently large $d$ ) are well separated (Fig. 8) and the total energy follows that of two identical dots each containing 4 electrons plus the Coulomb repulsion between two point-like charges $\left(4 e^{-}\right)$in distance $d$. However, besides the quantum mechanical phenomenon of delocalized bonding and antibonding states we find a (nonclassical) xc energy related effect leading to the spin-polarized ground state.

Yannouleas and Landman 10 find from unrestricted Hartree-Fock calculations electron localization in the form of electron puddles and Wigner supermolecules for a ratio $\lambda$ between 
the oscillator length and Bohr's radius of about 1.4. In contrast, (exact) Quantum Monte Carlo calculations 28 indicate a transition from the Fermi liquid to the Wigner crystal regime if $\lambda$ is increased beyond a critical value of 4 . Thus, our calculations performed for $\lambda \approx 2$ are obviously in the Fermi liquid regime.

\section{CHEMICAL POTENTIAL}

Although recent experiments succeeded in identifying molecular properties of coupled quantum dots like bonding and antibonding states 13 the most convenient comparison between theoretical and experimental results is based on the chemical potential of the system. The chemical potential is defined as difference of total energies $\mu(N)=E(N)-E(N-1)$ which can be measured by capacitance spectroscopy 6, 6.29.

As it turns out, the dependence of $N$ on $\mu$ (Coulomb staircase) exhibits features which depend on the coupling strength $\mathbf{6}, 1,11$. While in the strong coupling limit the capacitance spectrum of the QDM is similar to that of the single quantum dot, it exhibits small spacings between the chemical potentials for $N=1,2, N=3,4, N=5,6$ etc. for the weakly coupled QDM which correspond to the pairing of peaks observed in capacitance spectroscopy6. 6 . Explanations for this phenomenon provided in the literature 6 are based on states localized at the centers of the molecule and neglect quantum mechanical coupling. Using these assumptions we can easily estimate the dependence of $\mu$ on $N$ for our system: If the QDM contains an even number $N$ of electrons one may assume that $N / 2$ electrons are localized at each dot. The total energy of the artificial molecule $E_{\mathrm{M}}(N)$ can be estimated by the energy

of the single dots containing $N / 2$ electrons $\left(E_{\mathrm{D}}(N / 2)\right)$ and the Coulomb repulsion between the dots which is approximated as Coulomb energy between point-like charges

$$
E_{\mathrm{M}}(N)=2 E_{\mathrm{D}}\left(\frac{N}{2}\right)+\frac{e^{2}(N / 2)^{2}}{4 \pi \varepsilon \varepsilon_{0} d}, N \text { even. }
$$

For odd $N$ one assumes $(N+1) / 2$ electrons to be in one dot and $(N-1) / 2$ in the other one and the approximation for the total energy yields

$$
\begin{aligned}
E_{\mathrm{M}}(N)= & E_{\mathrm{D}}\left(\frac{N+1}{2}\right)+E_{\mathrm{D}}\left(\frac{N-1}{2}\right) \\
& +\frac{e^{2}(N+1)(N-1) / 4}{4 \pi \varepsilon \varepsilon_{0} d}, N \text { odd. }
\end{aligned}
$$

Using these equations we can calculate the chemical potential $\mu_{\mathrm{M}}(N)=E_{\mathrm{M}}(M)-E_{\mathrm{M}}(N-1)$ of the QDM for even and odd $N$

$$
\begin{gathered}
\mu_{\mathrm{M}}(N)=E_{\mathrm{D}}\left(\frac{N}{2}\right)-E_{\mathrm{D}}\left(\frac{N}{2}-1\right)+\frac{e^{2} N / 2}{4 \pi \varepsilon \varepsilon_{0} d}, N \text { even, } \\
\mu_{\mathrm{M}}(N)=E_{\mathrm{D}}\left(\frac{N+1}{2}\right)-E_{\mathrm{D}}\left(\frac{N-1}{2}\right)+\frac{e^{2}(N-1) / 2}{4 \pi \varepsilon \varepsilon_{0} d}, N \text { odd. }
\end{gathered}
$$

Consequently, the addition energies which are necessary to add a further electron are

$$
\Delta \mu_{\mathrm{M}}(N)=\frac{e^{2}}{4 \pi \varepsilon \varepsilon_{0} d}, N \text { even, }
$$




$$
\Delta \mu_{\mathrm{M}}(N)=\mu_{\mathrm{D}}\left(\frac{N+1}{2}\right)-\mu_{\mathrm{D}}\left(\frac{N-1}{2}\right), N \geq 3 \text { odd }
$$

These equations indicate that in a weakly coupled quantum dot with strongly localized wavefunctions the addition of an electron to a configuration with an even electron number is energetically more expensive as it corresponds to an addition of an electron to a single quantum dot. On the other hand, adding an electron to an odd number of particles is relatively cheap because the addition energy only depends on the interdot Coulomb repulsion which is relatively small for large $d$ and gives rise to the typical pairing of conductance peaks. With growing electron numbers this model fails as the increasing Coulomb repulsion caused by more extended charge distributions is not included. This effect destroys the splitting of the paired peaks.

A conceptional difficulty of this model is that it is based on localized states yielding an asymmetric charge distribution for odd electron numbers. In our SDFT calculations, however, the delocalized states also lead to the pairing of the conductance peaks in the weakcoupling regime (Fig. 9) while providing symmetric electron densities for all $N$. Therefore, we would like to emphasize, that the pairing of peaks does not depend on electron states localized at one center of the QDM, but can be caused by delocalized wavefunctions as well. In addition, delocalized states provide the advantage that it is not necessary to determine a transition from a coherent to an incoherent regime when the distance $d$ between the centers is continuously increased.

\section{CONCLUSIONS}

We have studied the ground-state properties of QDMs containing 2, 4, and 8 electrons as a function of the distance $d$ between the centers of the molecule using SDFT. This concept includes spin effects in addition to the classical Coulomb repulsion. The results obtained for 4 and 8 electrons are reliable over the whole range of $d$ and can be predicted from the single-particle spectrum of the noninteracting system for not too weak confinement. For 4 electrons we find a transition from a spin-polarized to a spin-unpolarized configuration with decreasing coupling strength. The ground state of the 8-electron QDM can be characterized by a spin-polarized ground state (2 parallel spins) for strong coupling and a spin-polarized configuration with 4 aligned spins in the weak-coupling limit. In contrast, the two-electron QDM is well described by SDFT only in the strong-coupling regime whereas for large $d$ a HL approach yields better results. Finally we have discussed the Coulomb staircase diagram for the weak and the strong coupling regime and identified their characteristic features considering the background of delocalized states in an artificial molecule.

This work was funded by Deutsche Forschungsgemeinschaft (Grants No. SFB 348 and Ro 522/16). 


\section{REFERENCES}

${ }^{1}$ M. Kastner, Physics Today, 46, 24 (1993)

${ }^{2}$ P. Zanardi and F. Rossi, Phys. Rev B 59, 8170 (1999)

${ }^{3}$ K. Imamura, Y. Sugiyama, Y. Nakata, S. Muto, and N. Yokoyama, Jpn. J. Appl. Phys. 34, 1445 (1995)

${ }^{4}$ G. Burkard, D. Loss, and D. P. DiVicenzo, Phys. Rev. B 59, 2070 (1999)

${ }^{5}$ D. G. Austing, T. Honda, K. Muraki, Y. Tokura, and S. Tarucha, Physica B 249-251, 206 (1998)

${ }^{6}$ F. R. Waugh, M. J. Berry, D. J. Mar, R. M. Westervelt, K. L. Campman, and A. C. Gossard, Phys. Rev. Lett. 75, 705 (1995)

${ }^{7}$ F. R. Waugh, M. J. Berry, C. H. Crouch, C. Livermore, D. J. Mar, R. M. Westervelt, K. L. Chapman and A. C. Gossard, Phys. Rev. B 53, 1413 (1996)

${ }^{8}$ H. Imamura, P. A. Maksym, and H. Aoki, Phys. Rev. B 59, 5817

${ }^{9}$ M. Rontani, F. Rossi, F. Manghi, and E. Molinari, Sol. State Comm. 112, 151 (1999)

${ }^{10}$ C. Yannouleas and Uzi Landman, Phys. Rev. Lett. 82, 5325 (1999)

${ }^{11}$ S. Nagaraja, J.-P. Leburton, and R. M. Martin, Phys. Rev. B 60, 8759 (1999)

12 A. Wensauer, Spinabhängige Grundzustandsrechnungen für asymmetrische Quantendots, Master thesis, Regensburg, 1999

${ }^{13}$ T. H. Oosterkamp, T. Fujisawa, W. G. van der Wiel, K. Ishibashi, R. V. Hijman, S. Tarucha, and L. P. Kouwenhoven, Nature 395, 873 (1998)

${ }^{14}$ P. Hohenberg and W. Kohn, Phys. Rev. 136, B 864 (1964)

${ }^{15}$ W. Kohn and L. J. Sham, Phys. Rev. 140, A 1133 (1965)

${ }^{16}$ U. von Barth and L. Hedin, J. Phys. C 5, 1629 (1972)

${ }^{17}$ M. Koskinen, M. Manninen, and S. M. Reimann, Phys. Rev. Lett. 79, 1389 (1997)

18 O. Steffens, U. Rössler, and M. Suhrke, Europhys. Lett. 44222 (1998)

${ }^{19}$ B. Tanatar and D. M. Ceperley, Phys. Rev. B 39, 5005 (1989)

${ }^{20}$ V. Blum, G. Lauritsch, J. A. Maruhn, and P.-G. Reinhard, J. Comp. Phys. 100, 264 (1992)

${ }^{21}$ P.-G. Reinhard, Private communication

${ }^{22}$ G. W. Bryant, Phys. Rev. B 48, 8024 (1993)

${ }^{23}$ M. Reed and B. Simon, IV: Analysis of Operators. Methods of Modern Mathematical Physics, Academic Press, New York, 1978

${ }^{24}$ I. S. Gradsteyn and I. M. Ryzhik, Academic Press, New York, 1980

${ }^{25}$ O. Steffens, Künstliche Atome im Magnetfeld, PhD thesis, Regensburg, 1999

${ }^{26}$ However, for $0.5 \mathrm{a}_{0}^{*} \leq d \leq 4.5 \mathrm{a}_{0}^{*}$ the situation is vice versa: The HL triplet state is lower in energy than the HL singlet state as a consequence of the weak external confinement potential in comparison to the relatively strong external potential in the hydrogen molecule.

27 The effect of self-interaction in DFT calculations strongly depends on the number of particles. In the case of small electron numbers (2 or 3 particles) it can cause deviations whereas for larger numbers it can be neglected.

${ }^{28}$ R. Egger, W. Häusler, C. H. Mak, and H. Grabert, Phys. Rev. Lett. 82, 3320 (1999); 83, 462(E) (1999)

${ }^{29}$ S. Tarucha, D. G. Austing, T. Honda, R. J. van der Haage, and L. P. Kouwenhoven, Phys. Rev. Lett. 77, 3613 (1996) 


\section{FIGURES}

FIG. 1. Model potential for a QDM. In this picture the interdot distance is $d=13 \mathrm{a}_{0}^{*}$ and the strength of the parabolic potential near the centers is $\hbar \omega_{0}=3 \mathrm{meV}$.

FIG. 2. Lowest levels in the spectrum of the noninteracting system as a function of the interdot distance $d$. The levels are classified as bonding and antibonding states with even and odd parity, respectively.

FIG. 3. Lowest KS levels for the 2-electron QDM as a function of the interdot distance $d$ (the 2 lowest levels are occupied). In the shaded area at $d \approx 4.5 \mathrm{a}_{0}^{*}$ SDFT calculations yield a transition from a spin-unpolarized to a spin-polarized ground state.

FIG. 4. Comparison between the total energies of the SDFT calculation $\left(E_{\text {SDFT }}^{ \pm}\right)$and of the Heitler-London approach $\left(E_{\mathrm{HL}}^{ \pm}\right)$for the 2-electron QDM. + (-) denotes singlet (triplet) energies.

FIG. 5. Lowest KS levels for the 4-electron QDM as a function of the interdot distance $d$ (the 4 lowest levels are occupied). In the shaded area at $d \approx 2 \mathrm{a}_{0}^{*}$ SDFT calculations yield a transition from a spin-polarized $\left(S_{z}=1\right)$ to a spin-unpolarized $\left(S_{z}=0\right)$ ground state. Inset: Ground-state energy of the 4-electron QDM (crosses). The solid line describes the asymptotics for large $d$ in a quasi-classical picture as a combination of the ground state energy of two single dots containing 2 electrons $\left(E_{\text {Dot }}^{(2)}=11.418 \mathrm{meV}\right)$ and the Coulomb repulsion between two point-like charges $\left(2 e^{-}\right)$ in distance $d$.

FIG. 6. Ground-state density of a 4-electron QDM for $d=10 \mathrm{a}_{0}^{*}$. The character of two uncoupled dots is emphasized by the localization of the density in the centers of the molecule.

FIG. 7. Lowest KS levels for the 8-electron QDM as a function of the interdot distance $d$ (the 8 lowest levels are occupied). In the shaded area at $d \approx 7.5 \mathrm{a}_{0}^{*}$ SDFT calculations yield a transition from a spin-polarized $\left(S_{z}=1\right)$ to a higher spin-polarized $\left(S_{z}=2\right)$ ground state. Inset: Ground-state energy of the 8-electron QDM (crosses). The solid line describes the asymptotics for large $d$ in a quasi-classical picture as a combination of the ground state energy of two single dots

containing 4 electrons $\left(E_{\text {Dot }}^{(4)}=41.064 \mathrm{meV}\right)$ and the Coulomb repulsion between two point-like charges $\left(4 e^{-}\right)$in distance $d$.

FIG. 8. Ground-state density of a 8-electron QDM for $d=10 \mathrm{a}_{0}^{*}$. The character of two uncoupled dots is emphasized by the localization of the density to the centers of the molecule.

FIG. 9. Coulomb staircase (derived from SDFT results) for the weak-coupling regime (a) $\left(d=10 \mathrm{a}_{0}^{*}\right)$, for the strong coupling regime (c) $\left(d=3 \mathrm{a}_{0}^{*}\right)$, and for the circular parabolic quantum $\operatorname{dot}(\mathrm{c})\left(d=0 \mathrm{a}_{0}^{*}\right)$. The shaded areas in picture (a) indicate the pairing of conductance peaks. For higher electron numbers the pairing splits as a consequence of increasing Coulomb repulsion caused by more extended charge distributions. 
This figure "fig1-5.gif" is available in "gif" format from: http://arxiv.org/ps/cond-mat/0004209v1 
This figure "fig6-9.gif" is available in "gif" format from: http://arxiv.org/ps/cond-mat/0004209v1 